\title{
DISEÑO E IMPLEMENTACIÓN DE UNA RED LAN PARA LA EMPRESA SOFTEL
}

AUTORES: $\quad$ Melisa Pérez Gómez ${ }^{1}$

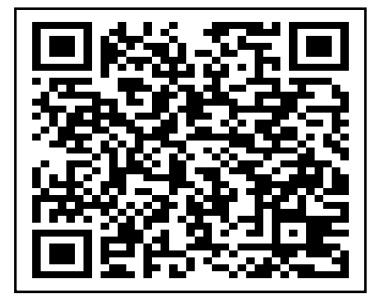

\section{DIRECCIÓN PARA CORRESPONDENCIA: melisa@,softel.cu}

Fecha de recepción: 01/02/2021

Fecha de aceptación: 21/06/2021

\section{RESUMEN}

Las telecomunicaciones son un campo de cambios rápidos: no ha terminado de surgir una tecnología cuando una alternativa más poderosa aparece en el horizonte. Estos cambios no son espontáneos sino el resultado de las presiones por la prestación de nuevos y mejores servicios. Las Redes de Área Local (LAN) se han convertido en un elemento estándar en las empresas, presionando inclusive la interconexión de redes locales y remotas. De otro lado han surgido nuevas aplicaciones y grandes bases de datos, que junto con aplicaciones de tiempo real como vídeo y multimedia, constituyen grandes consumidores de recursos de comunicaciones.

Ethernet ha sido durante mucho tiempo el estándar de-facto en Redes LAN. Su simplicidad y uso generalizado la han convertido en una tecnología muy popular en las redes de comunicaciones de datos actuales.

Por ello la Empresa de Soluciones Informáticas SOFTEL, pretende diseñar e implementar una red LAN y así mejorar la productividad de la misma aprovechando las potencialidades que ofrece dicha tecnología.

PALABRAS CLAVE: Ethernet; Red de computadoras; LAN.

\section{DESIGN AND IMPLEMENTATION OF A LAN NETWORK FOR SOFTEL ENTERPRISE}

\section{ABSTRACT}

Telecommunications is a fast-changing field: a technology has not finished emerging when a more powerful alternative appears on the horizon. These changes are not spontaneous but the result of pressures for the provision of new and better services. Local Area Networks (LAN) have become a standard element in companies, putting pressure on the interconnection of local and remote networks. On the other hand, new applications and large databases have emerged, which

\footnotetext{
${ }^{1}$ Ingeniera en Ciencias Informática, Estudiante de la Maestría en Informática Médica, Softel, Cuba. E-mail: melisa@softel.cu
} 
together with real-time applications such as video and multimedia, constitute large consumers of communication resources. Ethernet has long been the de-facto standard in LAN networks. Its simplicity and widespread use have made it a very popular technology in today's data communications networks. For this reason, the Company of Informatics Solutions SOFTEL intends to design and implement a LAN network and thus improve its productivity by taking advantage of the potential offered by that technology.

KEYWORDS: Ethernet; Computer Network; LAN.

\section{INTRODUCCIÓN}

Hoy en día, como nunca antes, el ser social necesita estar informado. Para estudiar problemas y tomas de decisiones es necesario disponer de datos precisos, en el lugar y en el instante preciso. En gran medida se logra lo anterior con las redes de computadoras.

Una red de computadoras no es más que un conjunto de computadoras interconectadas entre sí ocasional o permanentemente para compartir recursos de hardware y software.(1)

Existen varios tipos de redes con diferentes clasificaciones según su alcance geográfico; en este trabajo se presenta una propuesta de una red LAN con el fin de mejorar los servicios en la empresa SOFTEL y aprovechar las facilidades que brinda tener una red implementada.

Una red LAN (red de área local) es la interconexión de varias computadoras y periféricos. Su extensión está limitada físicamente a un edificio o a un entorno de 200 metros, o con repetidores podría llegar a la distancia de un campo de 1 kilómetro. Su aplicación más extendida es la interconexión de computadoras personales y estaciones de trabajo en oficinas, fábricas, entre otros, para compartir recursos e intercambiar datos y aplicaciones.(2)

\section{DESARROLLO}

En una empresa suelen existir muchos ordenadores, los cuales necesitan de su propia impresora para imprimir informes (redundancia de hardware), los datos almacenados en uno de los equipos es muy probable que sean necesarios en otro de los equipos de la empresa, por lo que será necesario copiarlos en este, pudiéndose producir desfases entre los datos de dos usuarios, la ocupación de los recursos de almacenamiento en disco se multiplican (redundancia de datos), los ordenadores que trabajen con los mismos datos tendrán que tener los mismos programas para manejar dichos datos (redundancia de software), entre otras situaciones.

La solución a estos problemas se resuelve implementando una red de área local LAN, esta permite compartir bases de datos (se elimina la redundancia de datos), programas (se elimina la redundancia de Software) y periféricos como puede ser un Módem, una Impresora, entre otros (se elimina la redundancia de Hardware); poniendo a nuestra disposición otros medios de comunicación como pueden ser el correo electrónico y el chat. Nos permite realizar un proceso distribuido, es decir, las tareas se pueden repartir en distintos nodos y nos permite la integración de los procesos y datos de cada uno de los usuarios en un sistema de trabajo corporativo. Tener la posibilidad de centralizar información o procedimientos facilita la administración y la gestión de los equipos.

130 UNESUM-Ciencias. Publicación cuatrimestral. Vol. 5, Año 2021, No. 4 (Número Especial) 
Además, una red de área local conlleva un importante ahorro, tanto de tiempo, ya que se logra gestión de la información y del trabajo, como de dinero, ya que no es preciso comprar muchos periféricos, se consume menos papel, y en una conexión a internet se puede utilizar una única conexión telefónica o de Banda Ancha compartida por varios ordenadores conectados en Red.(2)

Por todo lo anterior mencionado, se hace imprescindible implementar una red LAN en la empresa, para eso se ha realizado un diseño de una red LAN utilizando la siguiente metodología dividida por etapas:(1)

Etapa de estudio.

$>$ Etapa de diseño.

$>$ Etapa de elaboración de la solicitud de oferta y selección del vendedor.

$>$ Etapa de Instalación y puesta en Funcionamiento.

Etapa de análisis de las prestaciones y evaluación de los resultados.

\section{En la etapa de estudio:}

1) Determinar el número de estaciones de trabajo y su futura ubicación.

2) Definir cuáles serán los recursos compartidos.

3) Evaluar si es necesario cambiar el flujo de información de la organización.

4) Determinar que tareas seguirán realizándose localmente y cuáles pasarán a la red.

5) Conocer si se van a conectar en la red minicomputadores y/o mainframes.

6) Realizar un cálculo aproximado del costo de la red a partir de un conocimiento de los precios del mercado.

7) Estudiar los beneficios que reportaría la LAN ya partir de esto justificar su costo.

8) Elaborar un Informe a los Inversionistas.

La empresa de Soluciones informáticas SOFTEL, tiene como misión la informatización del sector de la salud en Cuba. Para esto cuenta con varios departamentos:

- Programación (se encuentra el personal encargado de programar las aplicaciones y cuenta con 26 dispositivos físicos en la red)

- Calidad (en este departamento labora el personal encargado de realizar pruebas de calidad a los productos que se desarrollan en la empresa, para eso tienen $\mathbf{2 0}$ dispositivos físicos a conectar en la red)

- Servicios Informáticos (son los que trabajan directamente con el cliente, en la implementación y adiestramiento de los mismos en las aplicaciones, para ello cuenta con 14 dispositivos físicos conectados en la red) 
- Minería de Datos (realizan los análisis de datos y optimizan códigos, para ello cuentan con 10 dispositivos físicos conectados en la red)

- Comercial (encargados de buscar y contratar clientes. Cuentan con 14 dispositivos físicos conectados en la red)

- Nodo (son los proveedores de servicios dentro de la red LAN y cuenta con 3 puestos de trabajo, además de los servidores, en total contará en total con $\mathbf{2 6}$ dispositivos físicos conectados en la red )

- Economía (encargados de toda la economía que se lleva en la empresa, los medios básicos y cuenta con 8 dispositivos físicos conectados en la red)

- Recursos Humanos (encargados de las nóminas de los trabajadores, pago de los trabajadores, vacaciones de los trabajadores, entre otras funciones y cuenta con 5 dispositivos físicos conectados en la red)

- Dirección General (es la persona que dirige la empresa, conoce las líneas de estrategia a seguir, entre otras funciones y cuenta con $\mathbf{4}$ dispositivos físicos conectados en la red)

- Recepción (es la encargada de recibir y orientar a cualquier persona ajena a la empresa y cuenta con 1 dispositivos físicos conectados en la red)

- Transporte (en este local se encuentra el personal que labora en el almacén y los encargados del transporte de la empresa, cuenta con $\mathbf{4}$ dispositivos físicos conectados en la red)

Además, se instalará una red Wifi, para conectar los dispositivos móviles a la red de la empresa. La misma se configurará en una subred solo para ella, para una mejor administración de la misma.

Para entender mejor se muestra una tabla de resumen de los recursos que se van a compartir en la red:

Tabla 1: Cantidad de recursos que se van a compartir por la red:

\begin{tabular}{|l|c|l|}
\hline Departamento & Cantidad de recursos & Número de Puntos de Red \\
\hline Programación & -1 impresora & $\begin{array}{l}27(1 \text { impresora compartida }+ \\
\left.25 \mathrm{Pc}^{\prime} \mathrm{s}+1 \text { punto extra }\right)\end{array}$ \\
\hline Calidad & $-25 \mathrm{Pc}^{\prime} \mathrm{s}$ & $\begin{array}{l}20(1 \mathrm{impresora} \text { compartida }+ \\
18 \mathrm{Pc} \text { 's }+1 \text { punto extra })\end{array}$ \\
\hline Servicios Informáticos & $-18 \mathrm{Pc}^{\prime} \mathrm{s}$ & $\begin{array}{l}25(2 \text { impresoras }+6 \text { puntos de } \\
\text { red físico }+ \text { dispositivos } \\
\text { móviles para conectar por wifi. }\end{array}$ \\
& -5 Pc's & $\begin{array}{l}10(1 \text { impresora }+8 \text { Pc's }+1 \\
\text { punto extra })\end{array}$ \\
\hline Minería de Datos & -15 Laptos & 1 impresora \\
& -8 Pc's & \\
\hline
\end{tabular}

132 UNESUM-Ciencias. Publicación cuatrimestral. Vol. 5, Año 2021, No. 4 (Número Especial) 


\begin{tabular}{|c|c|c|}
\hline Nodo & $\begin{array}{ll}- & 1 \text { impresora } \\
- & 3 \mathrm{PC}^{\prime} \mathrm{s} \\
- & 4 \text { Servidores Físicos } \\
\text { (todos los demás } \\
\text { virtualizados) } \\
\text { - } 1 \text { AP para las } \\
\text { conexiones Wifi }\end{array}$ & $\begin{array}{l}26 \text { puntos (1 impresora }+3 \\
\text { PC's }+1 \mathrm{AP}+21 \text { tarjetas de } \\
\text { red de los servidores })\end{array}$ \\
\hline Economía & $\begin{array}{l}\text { - } 1 \text { impresora } \\
\text { - } 7 \text { Pc's }^{\prime}\end{array}$ & $\begin{array}{l}8 \text { (1 impresora }+7 \mathrm{Pc}^{\prime} \mathrm{s}+1 \\
\text { punto extra })\end{array}$ \\
\hline Recursos Humanos & $\begin{array}{l}\text { - } 1 \text { impresora } \\
\text { - } 4 \text { Pc's }^{\prime}\end{array}$ & 5 (1 impresora +4 Pc's $)$ \\
\hline Dirección General & $\begin{array}{l}-2 \text { impresoras } \\
-\quad 2 \text { Pc's }^{\prime}\end{array}$ & $\begin{array}{l}5 \text { (2 impresoras }+2 \mathrm{Pc}^{\prime} \mathrm{s}+1 \\
\text { punto extra) }\end{array}$ \\
\hline Transporte & $\begin{array}{l}\text { - } 1 \text { impresora } \\
\text { - } \quad 3 \text { PC's }^{\prime}\end{array}$ & $4\left(1\right.$ impresora +3 Pc's $\left.^{\prime}\right)$ \\
\hline Recepción & $-1 \mathrm{Pc}$ & $2\left(1 \mathrm{Pc}^{\prime} \mathrm{s}+1\right.$ punto extra) \\
\hline \multicolumn{2}{|c|}{ Total de puntos físicos de Red } & $\begin{array}{l}132 \text { puntos y } 120 \text { el resto se } \\
\text { conectará por wifi }\end{array}$ \\
\hline
\end{tabular}

En el punto 3 de esta etapa, se cambiará totalmente el flujo de trabajo, porque antes de implementar la red, toda la información se compartía a nivel de memoria, ahora con la implementación de una Red LAN, se podrá aprovechar todos los beneficios que esta tare consigo (Correo, Chat interno, Navegación nacional e internacional, DNS, entre otros).

En el punto 4, prácticamente todos los procesos se realizarán mediante la red, excepto la documentación secreta de la empresa, la cual tiene una máquina asignada ubicada en la dirección general, donde solo pueden acceder a ella de manera local, el personal autorizado.

Referente al punto 6 , se muestra en la tabla 2, un cálculo aproximado de los costos de la implementación de una red LAN:

Tabla 2: Cálculos aproximados de la implementación de la red LAN

\begin{tabular}{|l|l|l|l|l|}
\hline Dispositivos/Periféricos & Cantidad & Descripción & $\begin{array}{l}\text { Costo } \\
\text { unidad }\end{array}$ & $\begin{array}{l}\text { Total } \\
\text { (usd) }\end{array}$ \\
\hline Servidor & 2 & $\begin{array}{l}\text { Intel Xeon E5 (64 GB de } \\
\text { RAM, 4 x 4TB de Disco } \\
\text { duro) }\end{array}$ & 4500.00 \\
\hline
\end{tabular}




\begin{tabular}{|c|c|c|c|c|}
\hline $\begin{array}{lll}\text { Router } & \text { Huawei } & 4 \\
\text { puertos } & & \\
\end{array}$ & 1 & $\begin{array}{lr}\text { Dispositivo } & \text { de } \\
\text { interconexión externo e } \\
\text { interno con la IP real que } \\
\text { otorga etecsa para la } \\
\text { conectividad. }\end{array}$ & $246.00(3)$ & 246.00 \\
\hline ADSL (8 mb/seg) & 1 & $\begin{array}{l}\text { Servicio de internet } \\
\text { contratado con Etecsa }\end{array}$ & $\begin{array}{l}5565 * 7 \\
\text { (mensual) }\end{array}$ & $\begin{array}{l}467460 \\
\mathrm{MN}\end{array}$ \\
\hline $\begin{array}{l}\text { Switch } 24 \text { puertos Capa } \\
3 \text { de } 1 \text { GigaBit }\end{array}$ & 6 & $\begin{array}{l}\text { Dispositivos de } \\
\text { interconexión interno para } \\
\text { segmentar la red. }\end{array}$ & $89.00(4)$ & 534.00 \\
\hline Bobinas UTP cat 6 & 10 & $\begin{array}{l}\text { Son las cajas de cable UTP } \\
\text { cat } 6 \text { para preparar los } \\
\text { cables de red. Cada caja } \\
\text { tiene } 300 \text { metros. }\end{array}$ & $190.99(5)$ & 1909.9 \\
\hline $\begin{array}{l}\text { Access Point TP-Link } \\
\text { de } 300 \mathrm{Mbps}\end{array}$ & 1 & $\begin{array}{l}\text { Para implementar una red } \\
\text { wifi como extensión de la } \\
\text { LAN para dispositivos } \\
\text { móviles y portátiles }\end{array}$ & $64.00(6)$ & 64.00 \\
\hline $\begin{array}{l}\text { Caja de conectores Rj45 } \\
\text { (dobles) }\end{array}$ & 65 & $\begin{array}{l}\text { Son las cajitas que van a la } \\
\text { pared para conectar los } \\
\text { cables de red. Normalmente } \\
\text { dan la posibilidad de hacer } \\
\text { dos conexiones. }\end{array}$ & $9.72(7)$ & 631.80 \\
\hline $\begin{array}{l}\text { Cajas de Conectores } \\
\text { Rj45 (cada caja tiene } \\
300 \text { conectores) }\end{array}$ & 3 & $\begin{array}{l}\text { Son las puntas que se ponen } \\
\text { en los cables. }\end{array}$ & $15.99(8)$ & 47.97 \\
\hline Gabinete & 3 & $\begin{array}{l}\text { Son las cajas contenedoras } \\
\text { donde se instalan los } \\
\text { dispositivos de redes y } \\
\text { cables para protegerlos del } \\
\text { acceso de los usuarios. }\end{array}$ & $130(9)$ & 390.00 \\
\hline $\begin{array}{l}\text { Tarjetas LAN para } \\
\text { servidores }\end{array}$ & 15 & $\begin{array}{l}\text { Tarjetas de red adicionales } \\
\text { que se necesitarán para las } \\
\text { diferentes interfaces de } \\
\text { redes que administren los } \\
\text { servidores. }\end{array}$ & $45(10)$ & 675.00 \\
\hline \multicolumn{4}{|l|}{ Total } & $\begin{array}{l}8998.67 \\
\text { USD } \\
467460 \\
M N\end{array}$ \\
\hline
\end{tabular}

134 UNESUM-Ciencias. Publicación cuatrimestral. Vol. 5, Año 2021, No. 4 (Número Especial) 
Como se muestra en la tabla 2, implementar una red LAN es bien costosa para cualquier empresa, todo está en dependencia de la tecnología a emplear. En esta tabla se hace un estimado según los precios de algunos sitios que venden tecnología informática, donde se combinaron dos factores que se consideran determinantes en la adopción de cualquier tecnología: precio - calidad, ya que SOFTEL es una empresa desarrolladora de software, y aunque no se puede disponer de la tecnología más actualizada en temas de infraestructura, la empresa cuenta con un presupuesto para invertir y poder implementar una red LAN para aprovechar los beneficios que tiene la implementación de la misma:

- Facilidad para compartir los recursos informáticos.

- Aumenta la velocidad de transmisión de la información.

- Ahorro de tiempo (al no duplicar procesos) y recursos (impresoras, hojas, gastos de teléfono, entre otros).

- Alta fiabilidad y aumento en la seguridad de los datos.

- Posibilidad de expansión en caso de requerirlo.

- Gestión centralizada.

- Establecer políticas en todos los ordenadores.(11)

Con todos esos beneficios mencionados, se considera que aumentará la productividad de la empresa y la calidad de sus productos.

En correspondencia al punto 8, Realizar un Informe a los Inversionistas: se presenta el diseño de la red y posteriormente se va explicando los demás puntos de las siguientes etapas:

La edificación donde radica la empresa es bien sencilla, es de una sola planta que tiene de largo aproximadamente 30 metros y de ancho 20 metros. En la siguiente imagen se muestra el plano de la empresa y como están ubicadas sus oficinas: 
Figura 1: Plano de Softel:

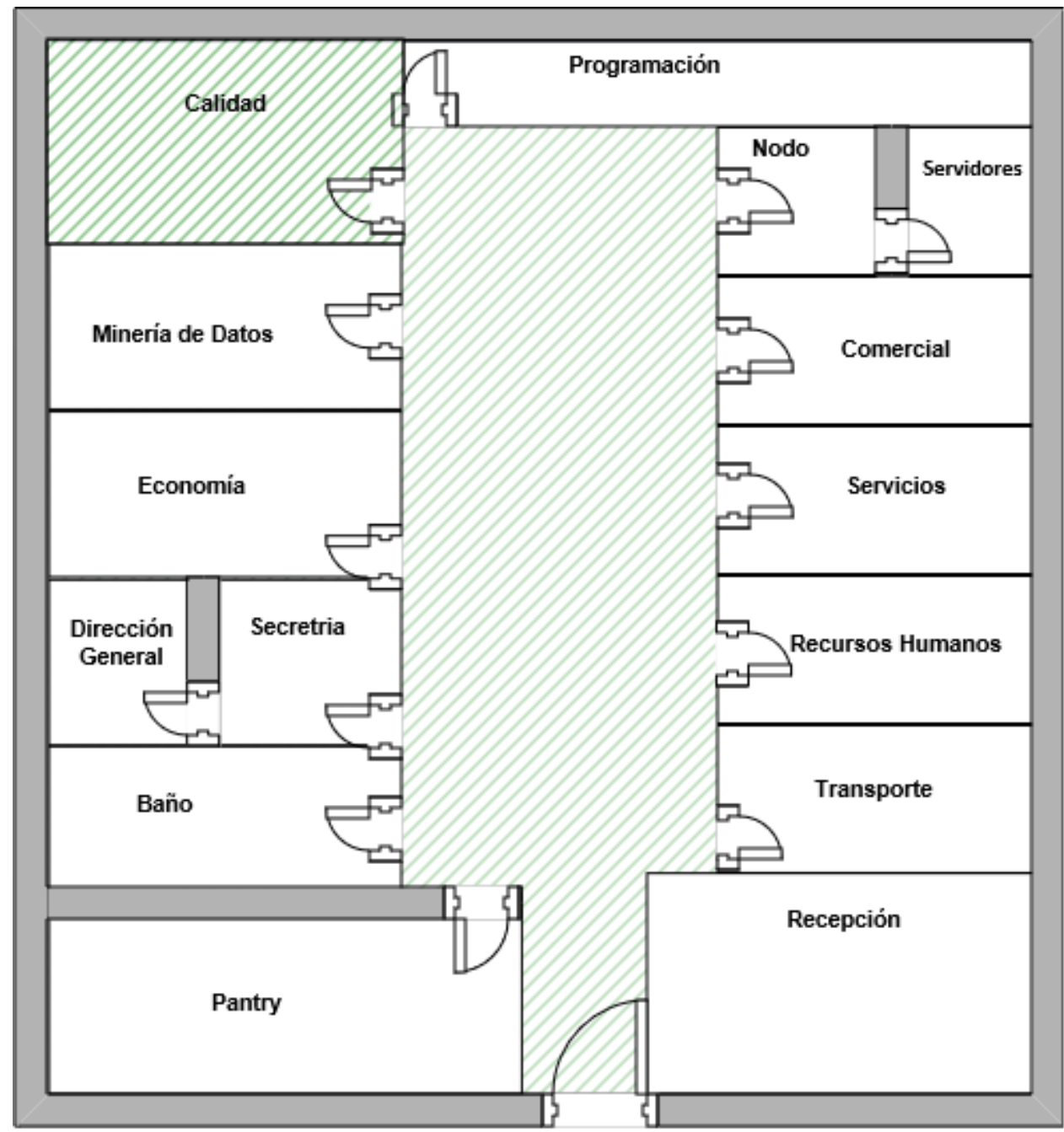

En el nodo se instalará el Rack de Comunicaciones que unirá todos los switches de todos los locales. El mismo tendrá 2 Switches de 24 puertos cada uno, todos los cables tendrán una etiqueta que identificará la conexión del cable (es decir si es de switch a switch o un dispositivo); la conexión de switch a switch siempre se colocará en el extremo izquierdo, es decir en los primeros puntos de cada switch.

El cableado irá por el falso techo, desde el nodo hasta las oficinas, y desde aquí irán en canaletas paralelas de manera primero vertical (hacerlas accesibles del techo hacia un punto a la pared, y de la pared se extenderá de manera horizontal por cada puesto de trabajo que necesite un punto de conexión).

Por otro lado, se dispondrán de tres gabinetes, uno de ellos estará instalado en el local de Calidad (el cual tendrá las conexiones de los locales de Calidad, Programación y Minería de Datos), el otro gabinete estará ubicado en el local de Comercial (contendrá las conexiones de los departamentos de Comercial, Servicio, Recursos Humanos y Transporte) y el último gabinete

136 UNESUM-Ciencias. Publicación cuatrimestral. Vol. 5, Año 2021, No. 4 (Número Especial) 
estará instalado en el local de Economía (que contendrá las conexiones de Economía, Dirección General y Recepción).

Para entender mejor como se pretende instalar la red LAN en la empresa, se muestra el diagrama de red en la Figura 2:

Figura 2: Diagrama de la red LAN de la empresa SOFTEL.

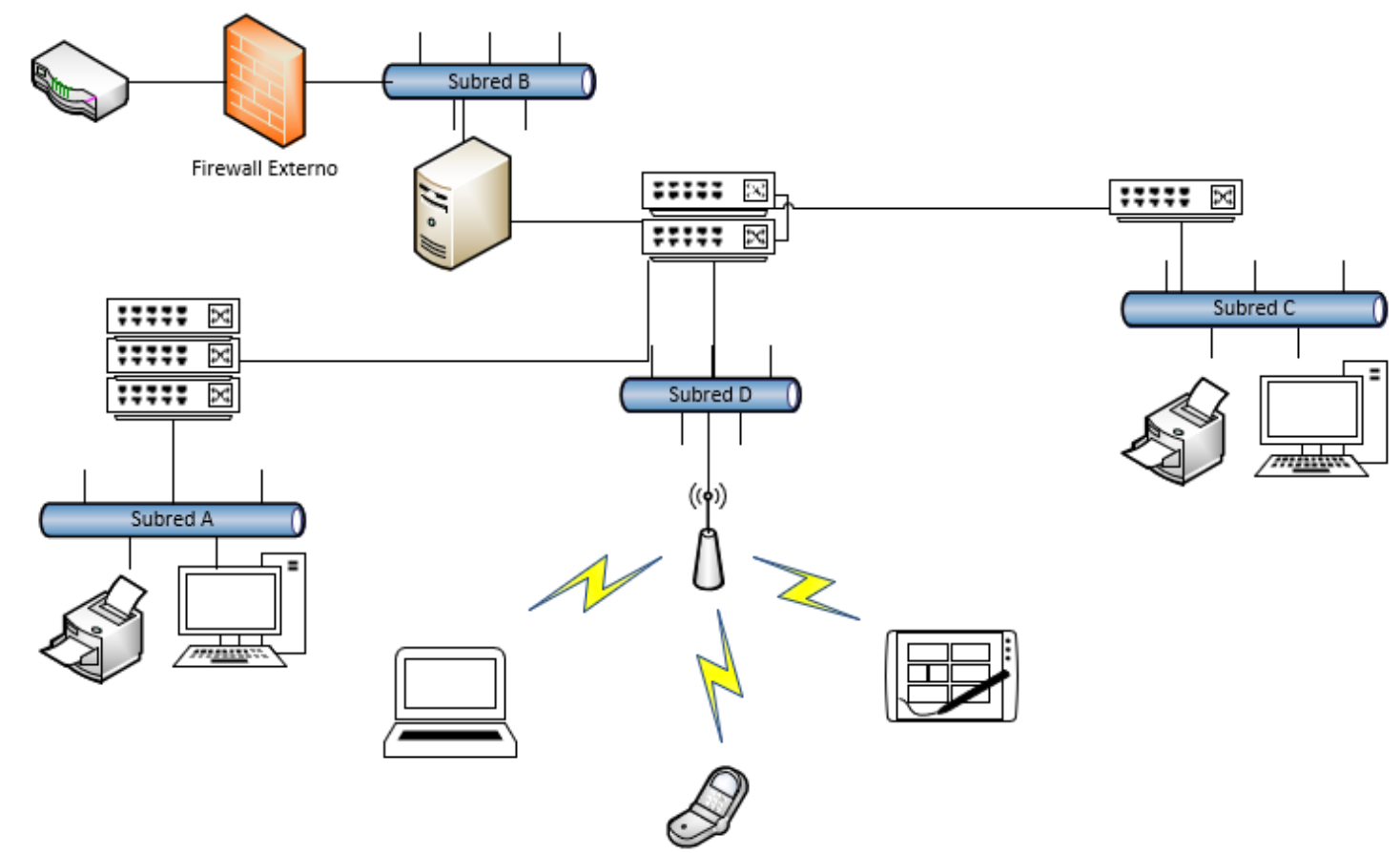

Donde la subred A, incluye los departamentos de: Calidad, Minería de Datos, Programación, Servicios y Comercial; mientras que la subred B incluye al nodo y sus conexiones. La subred C contiene las conexiones de los departamentos de Economía, Recursos Humanos, Dirección General, Transporte y Recepción.

La subred D, es la conexión Wifi que tendrá la empresa y el AP (Punto de Acceso) está instalado en las afueras del Departamento del Nodo.

La cantidad de máquinas y las distribuciones de las subredes se explican más adelante en la Etapa de Instalación y Puesta en funcionamiento.

\section{En la etapa de diseño:}

9) Seleccionar la(s) topologías) y norma(s) de red a emplear.

10) Seleccionar el soporte de transmisión a utilizar.

11) Seleccionar el Sistema Operativo de red que se usará.

12) Analizar la necesidad de emplear técnicas de conectividad. 
13) Considerar ampliaciones futuras de la red.

14) Realizar una evaluación primaria del tráfico.

15) Contemplar las necesidades del personal involucrado en la red.

16) Modificar, de ser necesario, el flujo de la información y seleccionar el software de aplicación.

Para la implementación de esta red LAN, se utilizará la topología estrella, porque dada su transmisión, una red en estrella activa tiene un nodo central activo que normalmente tiene los medios para prevenir problemas relacionados con el eco.

Además, la topología estrella se utiliza sobre todo para redes locales. La mayoría de las redes de área local que tienen un enrutador (router), un conmutador (switch) o un concentrador (hub) siguen esta topología. El nodo central en estas sería el enrutador, el conmutador o el concentrador, por el que pasan todos los paquetes.(12)

Como se muestra en la Figura 3 el funcionamiento de una red LAN con topología estrella:

Figura 3: Ejemplo de una red LAN con topología estrella.

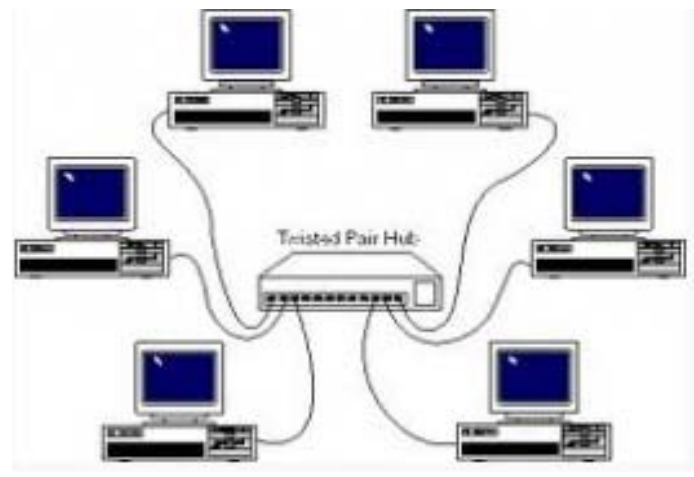

Como se observa en la figura 1, con una topología estrella aseguramos:

- Tiene los medios para prevenir problemas.

- Si una PC se desconecta o se rompe el cable, solo queda fuera de la red esa PC.

- Fácil de agregar, reconfigurar la arquitectura PC.

- Fácil de prevenir daños o conflictos.

- Permite que todos los nodos se comuniquen entre sí de manera conveniente.

- El mantenimiento resulta más económico y fácil que la topología bus.

Referente al punto 11, se utilizarán para la red LAN de la empresa tres sistemas operativos: Window Server 2012 R2, principalmente para el servidor de dominio, Debian 8 para otros servidores como proxy, correo, entre otros y para las estaciones de trabajo de los usuarios, se utilizarán Window 10.

138 UNESUM-Ciencias. Publicación cuatrimestral. Vol. 5, Año 2021, No. 4 (Número Especial) 
En los puntos 12 y 13 las técnicas de conexión serán las siguientes:

- Implementar dentro de la red LAN una red Wifi y

- Segmentar la red en subredes más pequeñas

Para asegurarnos en un futuro de una posible ampliación en cuanto a números de dispositivos, se implementará una red LAN segmentada a su vez en VLAN (Virtual Local Area Network), es decir la red LAN estará segmentada en varias subredes que harán uso de los mismos servicios que estén configurados dentro de la misma.

Una VLAN no es más que es un segmento lógico más pequeño dentro de una gran red física cableada. Las diferentes estaciones se combinan en una solución de red independiente de su ubicación: siempre que estén conectadas entre sí en la misma LAN, es posible combinarlas mediante una VLAN.(13)

Las redes VLAN facilitan el diseño de una red para dar soporte a los objetivos de una organización. Además:

- Mejor rendimiento de la red, la división de las redes planas de capa 2 en varios grupos de trabajo lógicos (dominios de difusión) reduce el tráfico innecesario en la red y mejora el rendimiento.

- Permite aplicar medidas de seguridad más potentes. Si queremos evitar que personas no autorizadas accedan a datos confidenciales, es una buena idea limitar la red a un grupo pequeño. Con una VLAN, el dominio de broadcast está limitado a unas pocas estaciones. De esta forma, la difusión o broadcast no puede llegar a personas a las que no esté dirigida la información.

- También son prácticas para aislar fallos de dominio y, por tanto, reducir el tiempo medio que se tarda en detectar y resolver el problema.

- Mayor flexibilidad ante cambios futuros.(11)

- Reducir costos, poca necesidad de actualizaciones de red costosas y al uso más eficaz de los enlaces y del ancho de banda existentes.

- Mayor eficiencia del personal de TI, las VLAN facilitan el manejo de la red debido a que los usuarios con requerimientos similares dered comparten la misma VLAN. Cuando se dispone de un switch nuevo, se implementan todas las políticas y los procedimientos que ya se configuraron para la VLAN específica cuando se asignan los puertos. También es fácil para el personal de TI identificar la función de una VLAN proporcionándole un nombre.(14)

Además, se implementará una subred wifi, para incorporar los dispositivos móviles y aprovechar las potencialidades de las redes wifi dentro de la red LAN:

- Reducción de costos, ya que se eliminan o se reducen los costos de cableado durante los traslados de oficina, nuevas configuraciones, reestructuraciones o expansiones. 
- Movilidad: Acceso a la red desde cualquier lugar en la empresa y con diferentes dispositivos. Hoy en día la mayoría de los dispositivos electrónicos como laptops, teléfonos, televisores, cámaras, impresoras y mucho más vienen con la tecnología wifi para conectarse fácil y rápidamente.

- El acceso a la información y a las aplicaciones clave de su empresa ayudan al equipo laboral a realizar su trabajo, aumentar su productividad y fomentar la colaboración.

- Los visitantes pueden tener acceso de invitado seguro a Internet y a sus datos de empresa.

- La red wifi proporciona protecciones de seguridad sólidas para que sus datos sólo estén disponibles para las personas a las que le permita el acceso.

- Mediante herramientas de softwares, la configuración y administración es de manera remota pudiendo monitorear todos los puntos de acceso wifi de las distintas sedes que tenga o no la empresa.(15)

\section{En la etapa de elaboración de la solicitud de oferta y selección del vendedor:}

17) Especificar características de los elementos a adquirir.

18) Realizar un análisis económico.

19) Analizar las características del vendedor.

Para implementar la red LAN se hizo un estudio de las tecnologías en el mercado, los precios de las mismas, la calidad de los productos según opiniones de usuarios de otras empresas que han utilizado una determinada tecnología, también se leyeron opiniones dadas en los sitios oficiales de dichas marcas (Dell, Fujitsu, Cisco, Huawei, Intel, entre otros), y como se mencionó anteriormente, se hizo un balance entre costos - calidad - beneficios de adquirir una determinada tecnología, para esto se determinó que la oferta más económica era la descrita anteriormente en el informe a los inversionistas:

Tabla 3: descripción de las tecnología seleccionada.

\begin{tabular}{|l|l|l|l|l|}
\hline $\begin{array}{l}\text { Dispositivos/Perifé } \\
\text { ricos }\end{array}$ & $\begin{array}{l}\text { Canti } \\
\text { dad }\end{array}$ & Descripción & $\begin{array}{l}\text { Costo } \\
\text { unidad }\end{array}$ & Total (usd) \\
\hline Servidor & 2 & $\begin{array}{l}\text { Intel Xeon E5 (64 GB de RAM, } \\
4 \text { x 4TB de Disco duro) }\end{array}$ & 2250.00 & 4500.00 \\
\hline $\begin{array}{l}\text { Router Huawei 4 } \\
\text { puertos }\end{array}$ & 1 & $\begin{array}{l}\text { Dispositivo de interconexión } \\
\text { externo e interno con la IP real } \\
\text { que otorga etecsa para la } \\
\text { conectividad. }\end{array}$ & $246.00(3)$ & 246.00 \\
\hline ADSL (8 mb/seg) & 1 & $\begin{array}{l}\text { Servicio de internet contratado } \\
\text { con Etecsa 5565*7 } \\
\text { (mensual) }\end{array}$ & $467460 \mathrm{MN}$ \\
\hline Switch Capa 3 & 6 & $\begin{array}{l}\text { Dispositivos de interconexión } \\
\text { interno para segmentar la red. }\end{array}$ & $89.00(4)$ & 534.00 \\
\hline
\end{tabular}

140 UNESUM-Ciencias. Publicación cuatrimestral. Vol. 5, Año 2021, No. 4 (Número Especial) 


\begin{tabular}{|l|l|l|l|l|}
\hline Bobinas UTP cat 6 & 10 & $\begin{array}{l}\text { Son las cajas de cable UTP cat 6 } \\
\text { para preparar los cables de red. } \\
\text { Cada caja tiene 300 metros. }\end{array}$ & $190.99(5)$ & 1909.9 \\
\hline $\begin{array}{l}\text { Access Point TP- } \\
\text { Link de 300 Mbps }\end{array}$ & 1 & $\begin{array}{l}\text { Para implementar una red wifi } \\
\text { como extensión de la LAN para } \\
\text { dispositivos móviles yortátiles }\end{array}$ & $64.00(6)$ & 64.00 \\
\hline $\begin{array}{l}\text { Caja de conectores } \\
\text { Rj45 (dobles) }\end{array}$ & 65 & $\begin{array}{l}\text { Son las cajitas que van a la pared } \\
\text { para conectar los cables de red. } \\
\text { Normalmente dan la posibilidad } \\
\text { de hacer dos conexiones. }\end{array}$ & 631.80 \\
\hline $\begin{array}{l}\text { Cajas de } \\
\text { Conectores Rj45 } \\
\text { (cada caja tiene } \\
300 \text { conectores) }\end{array}$ & 3 & $\begin{array}{l}\text { Son las puntas que se ponen en } \\
\text { los cables. 15.99(8) }\end{array}$ & 47.97 \\
\hline Gabinete & 3 & $\begin{array}{l}\text { Son las cajas contenedoras } \\
\text { donde se instalan los } \\
\text { dispositivos de redes y cables } \\
\text { para protegerlos del acceso de } \\
\text { los usuarios. 130(9) }\end{array}$ & 390.00 \\
\hline $\begin{array}{l}\text { Tarjetas LAN para } \\
\text { servidores }\end{array}$ & 15 & $\begin{array}{l}\text { Tarjetas de red adicionales que } \\
\text { se necesitarán para las diferentes } \\
\text { interfaces de redes que } \\
\text { administren los servidores. }\end{array}$ & $45(10)$ & 675.00 \\
\hline
\end{tabular}

La empresa cuenta con 3 UPS profesionales que aguantarán la carga de los 2 servidores profesionales ante una falta del fluido eléctrico, además como parte de una inversión, años atrás cuenta con un Panel de Parcheo (Patch Panel) que soporta hasta 13 unidades.

En el aspecto de los Software a emplear en las redes y las estaciones de trabajo se utilizarán básicamente tres softwares: Debian 7 para las aplicaciones que se trabajan en la empresa, Windows 2012 R2 para el servicio DNS (DomainNameService) y Active Directory (Directorio Activo), Proxmox para virtualizar, y en las estaciones de trabajo Windows 10 Enterprise, Office 2016, Adobe Reader 12, herramientas para desarrollar aplicaciones y manejar bases de datos como son Navicat, Netbeans, entre otras (dichas aplicaciones de desarrollo las usaran los programadores según sus preferencias).

A continuación, se hace un desglose de los servicios por servidores:

Tabla 4: Distribución de los servicios por servidores 


\begin{tabular}{|c|c|c|}
\hline Servidor & Sistema Operativo & Aplicaciones/Servicios \\
\hline 1 & Proxmox & $\begin{array}{l}\text { - } \quad \text { Windows Server } 2012 \text { R2: Directorio Activo. } \\
\text { - } \text { OPNSense: Firewall Interno. } \\
\text { - } \quad \text { Proxmox: para virtualizar máquinas que se } \\
\text { necesitan para las aplicaciones de la empresa. }\end{array}$ \\
\hline 2 & Proxmox & $\begin{array}{l}\text { Contiene las siguientes máquinas virutales: } \\
\text { - Zimbra: Correo Nacional e Internacional. } \\
\text { - } \quad \text { Squid: es una aplicación que funciona como proxy } \\
\text { para controlar la navegación de los usuarios dentro } \\
\text { de la red. } \\
\text { - Windows Server } 2012 \text { R2: servidor DNS }\end{array}$ \\
\hline $3 *$ & PFSense & $\begin{array}{l}\text { Firewall Externo y enrutador, natea la IP real que otorga } \\
\text { Etecsa para repartirlas en IP privadas dentro de la red. }\end{array}$ \\
\hline $4^{*}$ & Proxmox & $\begin{array}{l}\text { - Se tiene un sistema de salvas de los principales } \\
\text { servicios de la red creado, dichas salvas están } \\
\text { programadas que se realicen cada dos días en la } \\
\text { semana, las salvas serán acumulativas y } \\
\text { mensualmente se borrarán todas las salvas menos } \\
\text { las dos últimas (completa que tenga el sistema } \\
\text { guardado) }\end{array}$ \\
\hline
\end{tabular}

****Esta es una máquina convencional con la que contaba la empresa, Intel Core I3, con 2 GB de Ram y $500 \mathrm{~Gb}$ de Disco duro, convertida en servidor, solamente se utiliza como Firewall Externo y para natear la ips.

****Esta es una máquina convencional con la que contaba la empresa, Intel Core i5 con 4G de Ram y 2 TB de disco duro para almacenar las salvas programadas de los servicios de la red.

Ambas son Pc con las que cuenta la empresa, no hay que invertir dinero en ellas.

También se incluye el gasto de instalación del cableado de la red, en Cuba existen varias empresas que brindan este servicio, una de ellas es Copextel, la cual hizo una oferta que incluye, Instalación todo el cableado UTP de la red, e instalación y configuración de la PC que se utilizará como Firewall Externo y enrutador, todo esto por el precio de $20565.30 \mathrm{MN}$.

Además, se contrató con Copextel, el Servicio de Reparación y Reposición de Piezas por un costo anual de $2000.50 \mathrm{MN}$, el cual implica realizar un mantenimiento anual del $50 \%$ de las máquinas de la empresa (las que el cliente designe) y en caso de requerirlo reponer alguna pieza que se rompa (memoria RAM, Disco Duro, fuente, teclado, mouse, monitor y bocinas), siempre y cuando Copextel tenga la pieza en existencia. 
De menara general el gato de la inversión para implementar una red LAN es: 8998.67 USD + 20565.30 MN, ymensualmente entre los gastos de la empresa se incluye el pago de la conectividad: $\mathbf{4 6 7 4 6 0} \mathbf{M N}$.

\section{Etapa de instalación y puesta en funcionamiento:}

20) Instalar y probar el hardware.

21)Elaborar el registro de la red.

22) Organizar el trabajo en la red.

23) Realizar la prueba de todo el sistema.

24)Entrenar al personal vinculado a la red.

25)Establecer las normas y procedimientos.

26) Decidir cómo se realizará el período de transición al nuevo Sistema.

Una vez adquirida la tecnología, se comienza su instalación y configuración.

A continuación, se muestra como quedó organizada la LAN en subredes, según las necesidades de administración de los grupos:

\section{Direccionamiento de la red:}

Para el direccionamiento IP de la empresa y por la cantidad de dispositivos a conectar, la red principal será subdividida en subredes más pequeñas de manera lógica, es decir VLAN(Virtual Local Area Network) para una administración más clara de la red, como se muestra en la Figura 4:

Figura 4: Diagrama de la división de las subredes:

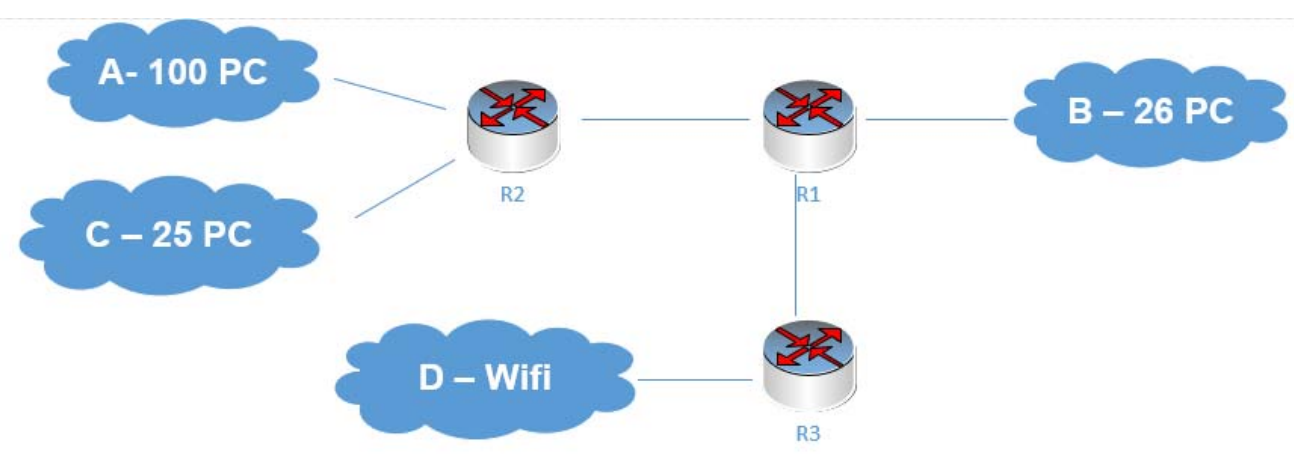

Leyenda:

- Subred A: contiene los departamentos de: Calidad, Minería de Datos, Programación, Servicios, Comercial.

- Subred B: contiene el departamento del Nodo con todos los servidores. 
- Subred C: contiene los departamentos de: Economía, Recursos Humanos, Mantenimiento, Dirección General, Recepción.

- Subred D, será la subred wifi, que contendrá el Access Point para que los dispositivos móviles se conecten a la wifi y adquieran los servicios por dicha red.

\section{Realizar la prueba de todo el sistema.}

Existen diferentes herramientas tanto de Hardware como de Software para la verificación del funcionamiento de una red LAN empresarial y para la identificación de posibles daños o errores que esta pueda tener.

Normalmente la mayoría de las fallas que se pueden presentar en una red LAN empresarial corresponden a los errores o daños físicos, estos errores vienen dados por problemas en el medio de transmisión. Para determinar problemas en el medio de transmisión el orden correcto es iniciar con una verificación física. Con esto se quiere decir que se debe verificar:

- Si el medio de transmisión es el apropiado.

- Si los conectores son los correctos y se encuentran correctamente ponchados.

- Si se encuentran correctamente conectados los equipos.

- De estar correctamente conectados si muestran link en la conexión.(16)

Tester de Red:Para la verificación de los cableados de cobres se utiliza un verificador o Tester de cableado, en este se puede realizar verificación del cableado sea coaxial o UTP. Con esta herramienta de Hardware se puede diagnosticar cual es el problema que presenta el cableado. Puede verificar si el cableado posee continuidad, se encuentra abierto, en cortocircuito o si el cable se encuentra con pares cruzados.

Desde el punto de vista del Software la herramienta empleada fue: Network View, muy útil la implementación de visores de red, estos nos muestran el esquema completo de nuestra red LAN, todos los equipos que se encuentran conectados y lo hace de manera gráfica lo cual nos ayuda a poder administrar y monitorear una red LAN empresarial, de esta manera podemos observar cuando se desconecta un equipo.

Luego de realizar pruebas de tráfico en la red, se determinó que el servicio que más consumía, era la concurrencia de todos los usuarios en internet, principalmente en los sitios de video como youtube.com, para evitar la latencia en la red y teniendo en cuenta que la internet forma parte de las herramientas empleadas por los departamentos que trabajan directo a la producción del software, el administrador de red ha establecido en los servidores: el archivo que se envía y recibe mediante el correo no podrá exceder los $7 \mathrm{Mb}$, referente a la navegación, según los grupos de las redes configurados: El grupo A dispondrá de 4 GB de navegación al mes, el grupo B de $7 \mathrm{~Gb}$ y los grupos $\mathrm{C}$ y $\mathrm{D}, 2 \mathrm{~Gb}$ respectivamente.

Una vez implementadas las reglas de navegación y correo, el tráfico de red mejoró sustancialmente. Aunque se sigue en el estudio de algunas herramientas de software libre para seguir supervisando y optimizando la red, de manera tal que todos los usuarios puedan hacer un debido uso de ella. 
Concluida la etapa de prueba de la red, comienza el Período de Adiestramiento de los usuarios en los servicios de la red, se llegó a la conclusión que los mismos eran usuarios avanzados, debido a que es una empresa desarrolladora de software, el $85 \%$ de la plantilla de la empresa son informáticos y por ende tienen un alto conocimiento del trabajo con la tecnología. Por eso se considera que el Período de Transición se realice de manera Directa.

Para finalizar esta etapa, se explican cómo están concebidos los siguientes Procedimientos que se necesita para implementar una red LAN:

- Procedimientos de Operación.

- Procedimientos de Mantenimiento: Este procedimiento está contratado con la empresa Copextel, el mismo establece que se le realizará mantenimiento al 50 por ciento de las máquinas de la empresa cada año, según las seleccionadas por el administrador de red, además incluye la reposición de piezas de repuesto según lo requiera, por ejemplo: memoria RAM, Monitor, Teclado, mouse, disco duro y bocinas.

- Procedimientos de copias: para este procedimiento se destinó una PC con la que contaba la empresa, que tiene $2 \mathrm{~Tb}$ de disco duro, el plan de salvas está configurado para que se realice semanalmente una acumulativa de los servicios de (correo, DNS, AD y Proxy), y una vez al mes se realice una integral. Todas se almacenarán en dicho servidor, y el administrador será el encargado de administrar las salvas que se dejan almacenas y las que se guardan en discos extraíbles.

- Procedimiento de reparación. este procedimiento lo tiene documentado la abogada de la empresa, el cual se hace mención en el contrato realizado con Copextel, empresa encargada de realizar la reparación y reposición (en caso de requerirlo) de los equipos informáticos adquiridos.

\section{Etapa de análisis de prestaciones y evaluación de resultados:}

27) Elaboración del Plan de Evaluación.

28) Determinar cuáles serán las herramientas a utilizar y los procedimientos a seguir para el análisis de la red.

29) Realizar la evaluación de prestaciones.

30) Realizar las modificaciones necesarias.

El Monitoreo de la red se considera fundamental para detectar defectos en la misma. Consiste en el uso de un sistema que constantemente monitoriza una red de ordenadores en busca de componentes defectuosos o lentos, para luego informar a los administradores de redes mediante correo electrónico, mensáfono (pager) u otras alarmas.(17)

Para realizar dicha acción los administradores de redes, utilizan herramientas que le facilitan dicha labor, en nuestro caso, inicialmente se utilizó Network View, pero posteriormente debido recomendaciones y a la búsqueda en internet de cual tendría mejores resultados, se decidió cambiar para Nagios. 
Nagios es un sistema de monitorización de redes de código abierto ampliamente utilizado, que vigila los equipos (hardware) y servicios (software) que se especifiquen, alertando cuando el comportamiento de los mismos no sea el deseado. Entre sus características principales figuran la monitorización de servicios de red (SMTP, POP3, HTTP, SNMP...), la monitorización de los recursos de sistemas hardware (carga del procesador, uso de los discos, memoria, estado de los puertos...), independencia de sistemas operativos, posibilidad de monitorización remota mediante túneles SSL cifrados o SSH, y la posibilidad de programar plugins específicos para nuevos sistemas.

Figura 5: Pantalla de los parámetros que mide Nagios.

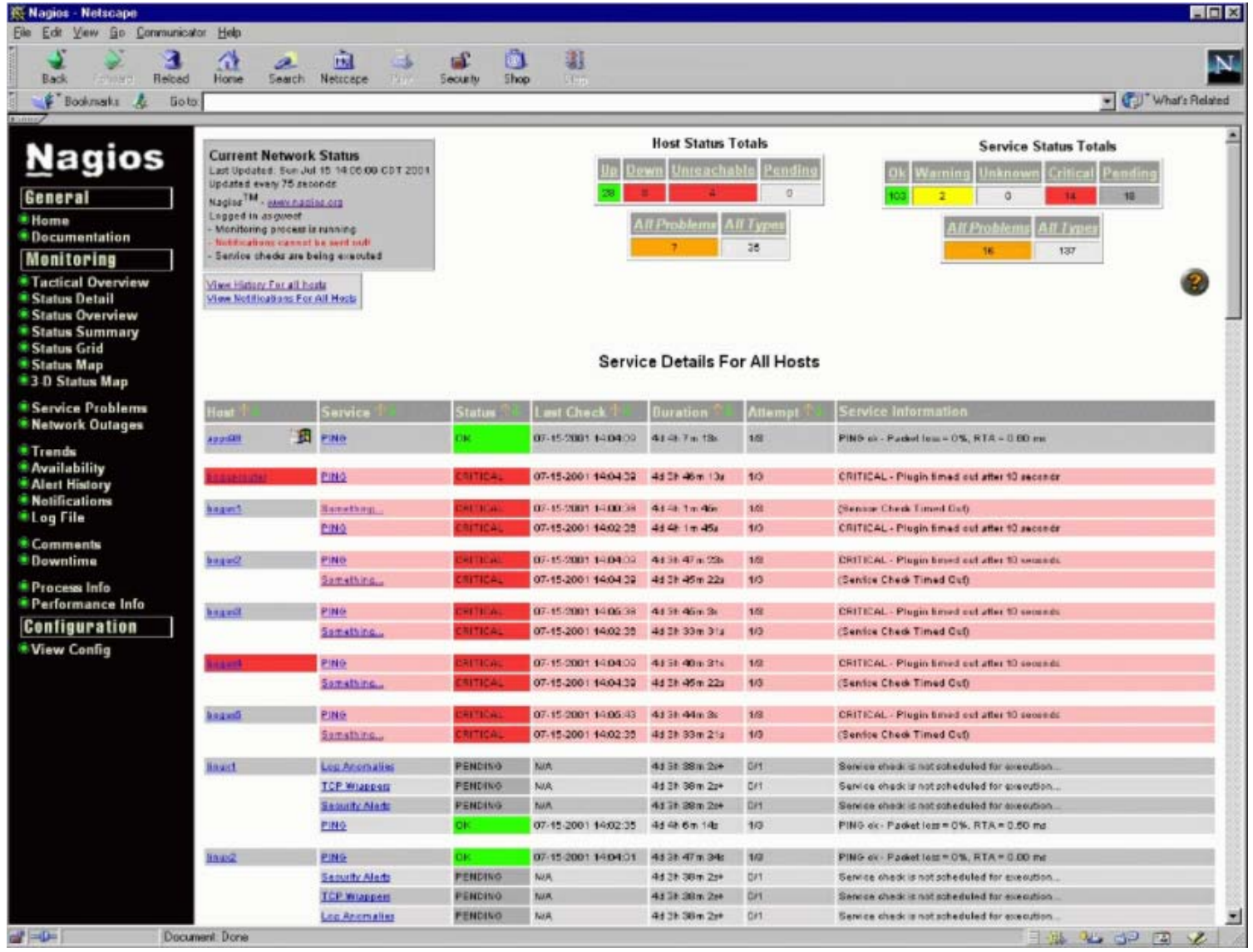

Se trata de un software que proporciona una gran versatilidad para consultar prácticamente cualquier parámetro de interés de un sistema, y genera alertas, que pueden ser recibidas por los responsables correspondientes mediante (entre otros medios) correo electrónico y mensajes SMS, cuando estos parámetros exceden de los márgenes definidos por el administrador de red.(18)

\section{CONCLUSIONES}

En el transcurso de este trabajo se identificaron varios de los dispositivos que conforman una red LAN, como la topología de red utilizada para la interconexión de los mismos. Además, se

146 UNESUM-Ciencias. Publicación cuatrimestral. Vol. 5, Año 2021, No. 4 (Número Especial) 
presentó una propuesta de inversión a tener en cuenta para comprar la tecnología necesaria para la implementación de la LAN en la empresa, teniendo en cuenta costos y calidad.

Se determinó que la empresa tiene usuarios avanzados, debido a ellos la Transición en el proceso podía ser de manera Directa.

También se logró evidenciar, que no es suficiente con tener la infraestructura implementada y configurada, sino que hay que darle seguimiento con herramientas de monitoreo de red, las cuales son imprescindible para detectar alguna anomalía en la misma, ya sea por algún dispositivo defectuoso o porque algunos de los servidores están muy cargados, y si es así habría que valorar la adquisición de uno nuevo, implementar un balanceo de cargas entre los mismos.

\section{REFERENCIAS BIBLIOGRÁFICAS}

1. MAR, O. 2021. Introducción a las redes de área local. In : . UCI, p. 50.

2. Red de área local (LAN) - EcuRed. [online]. [Accessed 15 June 2021]. Availablefrom: https://www.ecured.cu/Red_de_\%C3\%A1rea_local_(LAN)

3. AVM FRITZ!Box 7590 International - Modem Router 4x4 WiFi AC con MU-MIMO (1733 Mbps en $5 \mathrm{GHz}$ y $800 \mathrm{Mbps}$ en 2,4 GHz), Mesh, VDSL, ADSL2+, 1 x WAN Gigabit, 4 x LAN Gigabit, interfaz en Español: Avm: Amazon.es: Informática. [online]. [Accessed 26 June 2021]. Availablefrom: https://www.amazon.es/AVM-FRITZ-Box-7590International/dp/B074G5Z1PQ/ref=sr_1_4?dchild=1\&keywords=Modem+Ads1+Precio\&qid=16247 $26485 \& \mathrm{sr}=8-4$

4. Amazon.com: Tp-Link TL-SG1024D - Conmutador de escritorio Gigabit de 24 puertos - DW2697: Computers\&Accessories. [online]. [Accessed 15 June 2021]. Availablefrom: https://www.amazon.com/-/es/Tp-Link-TL-SG1024D-Conmutador-escritorioGigabit/dp/B003UWXFM0

5. Amazon.com: Nexxt - Cable UTP (Cat6, tipo $\mathrm{cm}$ ), color azul: Industrial \&Scientific. [online]. [Accessed 15 June 2021]. Availablefrom: https://www.amazon.com/-/es/Nexxt-Cable-Cat6-tipocolor/dp/B0812BSMBK

6. Access Point TP-LINK. [online]. [Accessed 26 June 2021]. Availablefrom: http://www.ds3comunicaciones.com/tplink/Access-Point.html

7. Amazon.com: Caja de conexiones RJ45 CAT6 con conector de red de 2 puertos, caja de extensión de cable de escritorio: Industrial \&Scientific. [online]. [Accessed 15 June 2021]. Availablefrom: https://www.amazon.com/-/es/conexiones-conector-puertos-extensi\%C3\%B3nescritorio/dp/B07MYNYLC3

8. Mr. Tronic Conectores RJ45 | 8P8C | Enchufe Modular (100 Unidades, CAT6 UTP): Amazon.es: Electrónica. [online]. [Accessed 15 June 2021]. Availablefrom: https://www.amazon.es/Mr-TronicConectores-Enchufe-Unidades/dp/B0859J7PTK?ref_=ast_sto_dp\&th=1\&psc $=1$

9. Gabinete Nexxt SKD 15U Instalable En Pared negro. Tienda CQNet [online]. [Accessed 26 June 2021]. Availablefrom: https://www.cqnetcr.com/gabinetes-racks/gabinete-nexxtskd-15u-instalable-en-pared-negro.html?fc=controllerComprar en Línea - NexxtSolutions Gabinete InfrastructureNexxtSolutionsSkd Armario Instalable En Pared negro RAL 9005-15U-19" 
10. Amazon.com: Tarjeta de red Dell PCI-E de 1 GB: Computers\&Accessories. [online]. [Accessed 26 June 2021]. Availablefrom: https://www.amazon.com/-/es/Tarjeta-red-Dell-PCIGB/dp/B00C1YESU6

11. Ventajas y límites de usar redes privadas virtuales. Escuela de Internet [online]. 28 September 2018. [Accessed 26 June 2021]. Availablefrom: https://www.escueladeinternet.com/ventajas-ylimites-de-usar-redes-privadas-virtuales/Qué es una VLAN, diferencias entre VLAN y LAN, cómo y cuándo utilizar redes privadas virtuales y ventajas e inconvenientes de hacerlo.

12. Red en estrella - EcuRed. [online]. [Accessed 15 June 2021]. Availablefrom: https://www.ecured.cu/Red_en_estrella

13. VLAN: ¿Qué es una Virtual Local Area Network? IONOS Digitalguide [online]. [Accessed 25 June 2021]. Availablefrom: https://www.ionos.es/digitalguide/servidores/knowhow/vlan/Con una VLAN podrás realizar una agrupación lógica dentro de tu red, sin tener que hacer recableado. ¿Cómo funciona y cuáles son las ventajas de una VLAN?

14. 3.1.1.2 Beneficios de las redes VLAN. [online]. [Accessed 26 June 2021]. Availablefrom: https://www.itesa.edu.mx/netacad/switching/course/module3/3.1.1.2/3.1.1.2.html

15. NETPRO. Beneficios de una Red Wifi Empresarial. Netpro [online]. 17 May 2018. [Accessed 26 June 2021]. Availablefrom: https://www.netpro.cl/beneficios-de-una-red-wifiempresarial/Las redes Wifi ofrecen innumerables ventajas para los espacios corporativos de cualquier tamaño físico u organizacional, entre los que destacan:

16. EDWIN ALFONSO GONZALEZ PIÑERES and HERNAN DARIO SERRANO MAYORGA. TECNICAS DE OPTIMIZACION Y MEJORAMIENTO DE UNA RED LAN EMPRESARIAL. [online]. CARTAGENA: UNIVERSIDAD TECNOLÓGICA DE BOLÍVAR, 2011. Availablefrom: https://biblioteca.utb.edu.co/notas/tesis/0061992.pdf

17. La importancia del software de monitoreo de red en una empresa. Computing [online]. 19 December 2018. [Accessed 4 July 2021]. Availablefrom: https://www.computing.es/infraestructuras/noticias/1 109344001801/importancia-del-software-demonitoreo-de-red-empresa.1.htmlLa monitorización de redes se considera ya fundamental en cualquier tipo de negocio.

18. NAGIOS: Herramienta para gestión-diagnóstico de Red en Linux - Euskadi+innova. [online]. 12 July 2007. [Accessed 4 July 2021]. Availablefrom: https://www.spri.eus/euskadinnova/es/enpresadigitala/agenda/nagios-herramienta-para-gestion-diagnostico-linux/3909.aspx 